\title{
Flood Detection Using Empirical Bayesian Networks
}

\author{
S.Rohini ${ }^{1}$, T.Hemalatha ${ }^{2}$ \\ ${ }^{1}$ Assistant Professor, Department of ECE, AITS, Tirupati, Andhra Pradesh, \\ ${ }^{2}$ Assistant Professor, Department of CS, SREC, Tirupati, Andhra Pradesh,
}

\begin{abstract}
Flood mapping from Synthetic Aperture Radar (SAR) data has attracted considerable attention in recent years. Flood is not only one of the widest spread natural disasters, which regularly causes large numbers of casualties with rising economic loss, extensive homelessness and disaster induced disease, but is also the most frequent disaster type. A valuable information source for such a procedure can be remote sensing synthetic aperture radar (SAR) imagery. However, flood scenarios are typical examples of complex situations in which different factors have to be considered to provide accurate and robust interpretation of the situation on the ground. For this reason, a data fusion approach of remote sensing data with ancillary information can be particularly useful. In this work, an Empirical Bayesian network is proposed to integrate remotely sensed data, such as multitemporal SAR intensity images and interferometric-SAR coherence data, with geomorphic and other ground information where as in the previous work the authors has used the Bayesian networks. The methodology is tested on a case study regarding a flood that occurred in the Visakhapatnam (India) on October 2014, monitored using a time series of TerraSAR-X data. It is shown that the synergetic use of different information layers can help to detect more precisely the areas affected by the flood, reducing false alarms and missed identifications which may affect algorithms based on data from a single source. The produced flood maps are compared to data obtained independently from the analysis of optical images; the comparison indicates that the proposed methodology is able to reliably follow the temporal evolution of the phenomenon, assigning high probability to areas most likely to be flooded, in spite of their heterogeneous temporal SAR/InSAR signatures, reaching accuracies of up to $89 \%$.
\end{abstract}

Keywords: Empirical Bayesian networks (BNs), data fusion, flood mapping, synthetic aperture radar (SAR) change detection, synthetic aperture radar (SAR)/interferometric SAR (InSAR) time series analysis.

\section{Introduction}

Accurate flood mapping is important for both planning activities during emergencies and as a support for the successive assessment of damaged areas .Flooding is a recurrent and dramatic natural disaster that affects several areas in the world, both in tropical and temperate regions, often causing great damages to lives and property, industrial settlements and infrastructures, and artistic and historical sites, aside from dramatically affecting local ecosystems. It is expected that the frequency of these phenomena will increase in the next future, due to climate change and the occurrence of several extreme weather events. Accurate knowledge of flood extents is crucial to improve disaster management and to mitigate the impact of flood episodes, during both the emergency phase, when an overall view is needed in order to plan relief efforts, and the aftermath, for the assessment of damaged areas.

Remote sensing data of affected areas, together with effective methods to exploit their information, can be particularly useful in the damage estimation phase: they offer a synoptic overview of wide areas, giving a snapshot of the extents of the flooding event [1]-[3]. Both optical and radar data have been used to infer information about the flooded areas [4]. Optical data have been used, particularly in urban areas [5], [6], where the effects of shadowing, layover, and foreshortening limit the results obtained by synthetic aperture radar (SAR) images, although new insight on these topics has been recently provided, e.g., in [3] and [7]. SAR sensors are particularly useful to produce flood maps, owing to their all weather and day night operation capability [8]-[10]. In particular, the latest generation of SAR sensors, such as TerraSAR-X and the COSMOSkyMed constellation, supplies a large amount of daily acquired radar images, characterized by high spatial resolution, that can be particularly useful in flood detection problems [3], [11], [12].

Nevertheless, flood scenarios are typical examples of complex situations in which different factors have to be considered to provide accurate and robust interpretation of the situation on the ground. The presence of many land cover types, each one with a particular signature in the presence of flood, requires modeling the behavior of different "objects" in the scene (not necessarily associated to land cover categories) in order to associate them to flood or no-flood conditions. In principle, a single SAR image acquired during an event could provide a flood map: its capability to detect inundations is based on the low backscatter response from smooth water covering the terrain, which allows to efficiently separate flooded areas from rougher nonflooded terrain that, instead, scatters the imping- ing signal in many different directions and produces a higher 
backscattering coefficient $\left(\sigma^{0}\right)$. However, many factors potentially complicate this simple situation. For instance, the contrast between dry soil and flooded one can be significantly reduced in the presence of wind roughening the water surface. Also, vertical structures on the ground, such as trees or man-made objects, could determine a double bounce mechanism of the radar signal, which is enhanced when the ground surface is flooded with respect to dry conditions: this can make the radar return from vegetated or urban flooded areas higher than that under nonflooded conditions [12].

In general, the exploitation of a set of images (as a multi- temporal stack) seems to provide more satisfactory results in the presence of these critical situations [8], [13]. Moreover, the interferometric SAR (InSAR) coherence information can be a viable means for detecting flooded areas as an extension of conventional change detection methods [14], [15]. However, the relative difficulty in planning pairs of SAR acquisitions suitable for InSAR use in coincidence with flood events, for legacy sensors, posed some problems in devising operational scenarios for the use of InSAR in flood monitoring. A renewed attention to this issue came with the availability of the aforementioned high-resolution sensors.

Empirical Bayes (EB) is a term that has many meanings, reflecting different approaches to solving problems. It can describe a methodology for both estimation and inference, an important distinction. In a majority of applications, statistical inference tends to be made using frequentist (classical) statistics. This is the type of inference that is applicable to a 'long-run' interpretation. That is, inference about a process is referred to a (usually imagined) sequence of replicated experiments. In contrast, the inference from Bayesian statistics is targeted to the data at hand. Each of these approaches has its strengths and weaknesses, and EB methods attempt to borrow the strengths from each approach.

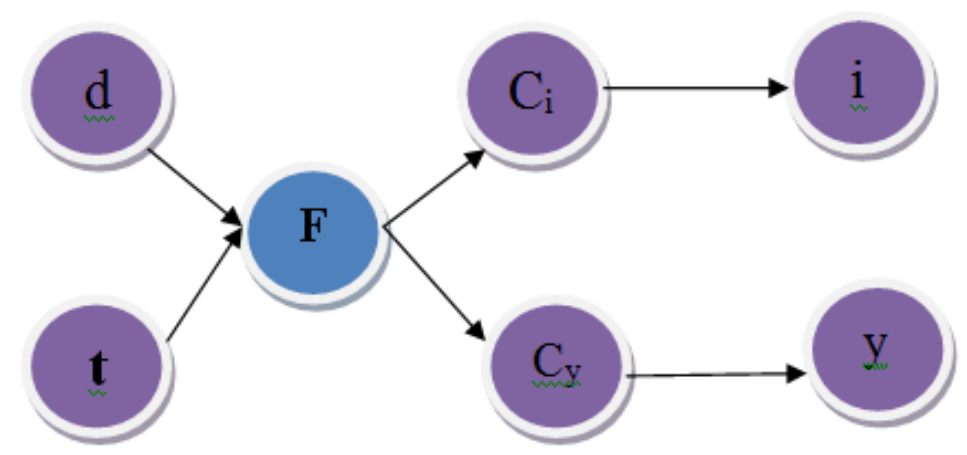

Figure 1. Bayes rule

This paper is organized as follows. In Section II, we discuss about the literature survey. In Section III, the proposed method is discussed in detail and some information on the study area and the considered flood event are reported, and the available data is described. In Section IV, we discuss about SAR data and TerraSAR$\mathrm{X}$ data. Finally, in Section V, conclusion is discussed..

\section{Literature Survey}

Recently, the combined analysis of multi-temporal SAR intensity and coherence trends has been proposed to detect flooded areas, reducing false alarms and missed identifications which affect algorithms based on SAR intensity alone [16], [17]. More generally, the fusion of multitemporal, multisensor, multiresolution, and/or multiplatform Earth observation image data, together with other ancillary information, seems to have a key role in the pursuit of a consistent interpretation of complex scenes: the decisions or actions taken considering data synergy exploitation are better (both qualitatively and quantitatively, in terms of accuracy, robustness, etc.) than would be possible if the sources were used individually [18]. In the case of flooding, distance from the river, terrain elevation, hydrologic informa- tion, or some combination thereof can add useful information that leads to a better performance in flood detection. In fact, the integration of remote sensing-derived flood extent maps and hydraulic models is now a promising research field (see, e.g., [19] and the references therein). In [20], a procedure was de- veloped in a geographic information system (GIS) environment in order to estimate the flooded area combining SAR imagery with ancillary information extracted from digital elevation topography. In [21], two different statistical methods have been used to integrate SAR data with high-precision topography and a river flow model. More recently, flood maps have been produced combining multitemporal SAR data and a hydrody- namic model by using a classification algorithm based on fuzzy logic [22]. SAR sensors are particularly useful to produce flood maps, owing to their all weather and day night operation capability [8]-[10]. In particular, the latest generation of SAR sensors, such as TerraSAR-X and the COSMO-SkyMed constellation, supplies a large amount of daily acquired radar images, characterized by high spatial resolution, that can be particularly useful in flood detection problems [3], [11], [12]. 
In recent years, BNs and EBNs and, more generally, probabilistic graphical models have been used as a data mining tool in many research fields, but rarely to process remote sensing data. In [25], a $\mathrm{BN}$ has been applied to two Landsat TM scenes acquired at different times on the same area, obtaining an overall change detection classification accuracy that overcomes the one obtained by other methods. However, to our knowledge, the capability of EBNs to join different kinds of data has been scarcely exploited. In [5], a simple probabilistic graphical model has been applied to estimate the state of roads during flooding, combining a DEM with satellite optical images: the obtained results outperform other methods based only on the exploitation of imagery data or only on DEM information. On the other hand, BNs and EBNs have been successfully used to construct risk assessment systems for natural disasters, such as rock falls, avalanches or wildfires: various kinds of data, such as land cover types extracted from the Corine Land Cover database, road and building densities, have been fused by means of EBNs to obtain reliable risk maps, although none included remotely sensed imagery.

In general, the exploitation of a set of images (as a multitemporal stack) seems to provide more satisfactory results in the presence of these critical situations [8], [13]. Moreover, the interferometric SAR (InSAR) coherence information can be a viable means for detecting flooded areas as an extension of conventional change detection methods [14], [15]. However, the relative difficulty in planning pairs of SAR acquisitions suitable for InSAR use in coincidence with flood events, for legacy sensors, posed some problems in devising operational scenarios for the use of InSAR in flood monitoring. A renewed attention to this issue came with the availability of the aforementioned high-resolution sensors.

\section{A . Bayesian Networks}

\section{Flood Map Production}

BNs combine probability theory and graph theory, introducing graph structures into a probabilistic model to represent dependence assumptions among the involved variables. One highly practical Bayesian learning method is the naive Bayes learner, often called the naive Bayes classifier. The interactions of the variables of a "system" are encoded through a directed acyclic graph (DAG) the nodes of the DAG represent the random variables, and the edges among them correspond to the direct influence of one node on another, modeled as conditional probabilities [23], [24]. The BN structure thus provides information about the underlying processes and the way that various variables communicate and share "information" as it spreads around the network. Formally speaking, the DAG encodes independence assumptions between variables.

$\mathrm{A} \mathrm{BN}$ is characterized by many attractive properties that can be particularly useful in modeling many real-world systems.

$$
P(A / B)=\frac{P(B / A) \cdot P(A)}{P(B)}
$$

Bayesian statistics is a branch of statistics that applies Bayes rule to solve inferential questions of interest where A represents unknowns and B represents data. Notations of the bayes or bayesian method are

$$
\begin{aligned}
& \quad \Theta \rightarrow \text { Model Parameters } \\
& \mathrm{y} \rightarrow \text { Data } \\
& \mathrm{y}^{\wedge} \rightarrow \text { Future Data }
\end{aligned}
$$

(1) y can be continuous or discrete

(2) $\theta$ can be a scalar or a vector

(3) $\mathrm{P}(\mathrm{u})$ represents the marginal pdf for $\mathrm{u}$.

(4) $\mathrm{P}(\mathrm{u} \mid \mathrm{v})$ represents the conditional pdf for $\mathrm{u}$ given $\mathrm{v}$

(5) $\mathrm{P}(\mathrm{u}, \mathrm{v})$ represents the joint pdf for $\mathrm{u}$ and $\mathrm{v}$.

Pdf--------Probability density function or probability mass function.

The parametric estimation of the bayesian networks is

For point or interval estimation of a parameter $\theta$ in a model $\mathrm{M}$ based on data $\mathrm{y}$, Bayesian inference is based off.[23]

Where,

$$
\begin{gathered}
\mathrm{P}(\theta \mid \mathrm{y})=\underset{\mathrm{P}(\mathrm{y} \mid \theta)}{\mathrm{P}(\mathrm{y})} \mathrm{p}(\theta) \\
\end{gathered}
$$

- $\mathrm{P}(\theta)$ is the prior density for the parameter

- $\mathrm{P}(\theta \mid \mathrm{y})$ is the posterior density for the parameter

- $\mathrm{P}(\mathrm{y} \mid \theta)$ is the statistical model ( or likelihood) and

- $\quad \mathrm{P}(\mathrm{y})$ is the prior predictive density (or marginal likelihood)

Bayesian prediction is based on a formulae: 
Where,

$$
\mathrm{P}(\hat{\mathrm{y}} \mid \mathrm{y})=\mathrm{p}(\hat{\mathrm{y}} \mid \theta) \cdot \mathrm{p}(\theta \mid \mathrm{y}) \cdot d \theta
$$

- $\quad \mathrm{p}(\theta \mid \mathrm{y})$ is the posterior

- $\quad \mathrm{p}(\hat{\mathrm{y}} \mid \theta)$ is the model

We consider here a BN designed as represented in Fig. 1: it combines information extracted by a set of images, and some ancillary information about the a priori spatial distribution of flood in the analyzed area. In detail, the random

$$
\begin{gathered}
\mathrm{P}\left(\mathrm{F}=\text { flood } \mid \mathrm{i}=\mathrm{i}^{*}, \gamma=\gamma^{*}, \mathrm{t}=\mathrm{t}^{*}, \mathrm{~d}=\mathrm{d}^{*}\right)=\begin{array}{l}
\mathrm{P}\left(\mathrm{F}=\text { flood, } \mathrm{i}=\mathrm{i}^{*}, \gamma=\gamma^{*}, \mathrm{t}=\mathrm{t}^{*}, \mathrm{~d}=\mathrm{d}^{*}\right) \\
\mathrm{P}\left(\mathrm{i}=\mathrm{i}^{*}, \gamma=\gamma^{*}, \mathrm{t}=\mathrm{t}^{*}, \mathrm{~d}=\mathrm{d}^{*}\right)
\end{array} \\
=\frac{\left[\sum_{\mathrm{Ct}} \mathrm{p}\left(\mathrm{i}=\mathrm{i}^{*} \mid \mathrm{C}_{\mathrm{i}}\right) \mathrm{p}\left(\mathrm{C}_{\mathrm{i}} \mid \mathrm{F}=\text { flood }\right)\right]\left[\sum \mathrm{C}_{\gamma} \mathrm{p}\left(\gamma=\gamma^{*} \mid \mathrm{C}_{\gamma}\right) \mathrm{p}\left(\mathrm{C}_{\gamma} \mid \mathrm{F}=\mathrm{flood}\right)\right] \mathrm{p}\left(\mathrm{F}=\text { flood } \mid \mathrm{t}=\mathrm{t}^{*}, \mathrm{~d}=\mathrm{d}^{*}\right)}{\sum_{\mathrm{F}}\left[\sum_{\mathrm{Ct}} \mathrm{p}\left(\mathrm{i}=\mathrm{i}^{*} \mid \mathrm{C}_{\mathrm{i}}\right) \mathrm{p}\left(\mathrm{C}_{\mathrm{i}} \mid \mathrm{F}\right)\right]\left[\mathrm{C}_{\gamma} \mathrm{p}\left(\gamma=\gamma^{*} \mid \mathrm{C}_{\gamma}\right) \mathrm{p}\left(\mathrm{C}_{\gamma} \mid \mathrm{F}\right)\right] \mathrm{p}\left(\mathrm{F} \mid \mathrm{t}=\mathrm{t}^{*}, \mathrm{~d}=\mathrm{d}^{*}\right)}
\end{gathered}
$$

variable $F$ is discrete and consists of only two states, flood and no flood, so that $P(F=$ flood $)=1-P(F=$ no flood). This is the variable that we want to estimate by statistical inference. The variable $i$ corresponds to the $n$ dimensional vector obtained from the SAR intensity imagery. Since, often, the flood state does not exhibit a simple causal relation with the imagery, due to the presence of particular types of land cover, particular backscattering mechanisms, etc., the intermediate variable class $\mathrm{Ci}$ is introduced [5]. It consists of $\mathrm{Ki}$ possible states that can be determined in two different ways: either by using some a priori knowledge on the scene or by extracting them automatically from the imagery. Anyway, the number $K i$ of possible states and the usefulness of the $C i$ variable are strongly dependent on the complexity of the analyzed scene. For example, if the area under observation is characterized only by unvegetated/barren areas and a single SAR intensity image is available, the variable $C i$ is unnecessary, and the $i$ random variable can be directly connected to $F$ : the backscatter values of flooded areas, in this case, will be very low and well separated from nonflooded ones. Otherwise, for more complex scenarios, such as in the presence of vegetated, forested, or urban areas, or if we want to follow the flood dynamics owing to the availability of multitemporal data, the introduction of the intermediate variable $\mathrm{Ci}$ allows to classify the various scattering behaviors into a finite number of types and then to focus the attention on the most interesting ones [5], [8]. The variable $\gamma$ corresponds to the $m$-dimensional vector obtained from the multitemporal InSAR coherence imagery. Similarly to the previous case, this variable is not directly connected with the $F$ one, and a hidden $C \gamma$ variable is introduced. It consists of $K \gamma$ possible states, for which the previous considerations hold. Finally, the variables $t$ and $d$ represent some ancillary information, i.e., a geomorphic flooding index (GFI) derived from a light-detectionand-ranging-derived DEM at 3-m posting, and the geometric distance from the river, properly modulated by the intensity of the considered event (the maximum expected distance reached by the river flood is estimated by considering the effective water level measurements along the river, available as readings from a gauge station), respectively. The $t$ variable is computed from the definition in [30] and [31] as follows:

$\mathrm{t}=\ln \left[\frac{\mathrm{h}_{\mathrm{r}}}{\mathrm{H}}\right]$

where $h_{r}$ is a function of the contributing area $A_{r}$ in the nearest section of the drainage network hydrologically connected to the point under exam and $H$ is the elevation difference between the cell under exam and the final point of the previously identified path.

In this work, we propose a statistical model able to perform flood detection by using information and data fusion. It is based on Empirical Bayesian networks (EBNs), one of the most common types of probabilistic graphical models combining probability theory and graph theory, which introduce graph structures into a probabilistic model to represent dependence assumptions among the involved variables . EBNs are a statistically well-founded method to combine imagery information with ancillary data, such as distance from the river, digital elevation models (DEMs), hydraulic models, etc.

\section{B. Empirical Bayesian Networks}

Consider the statistical model $\mathrm{p}(\mathrm{y} \mid \theta)$ with prior $\mathrm{p}(\theta)$ where our objective is the posterior distribution.[23] $\mathrm{p}(\theta \mid \mathrm{y}) \propto \mathrm{p}(\mathrm{y} \mid \theta) \cdot \mathrm{p}(\theta)$

Suppose $\mathrm{p}(\theta)=\mathrm{f}(\theta \mid \varphi)$ where $\mathrm{f}$ describes some family of distribution and $\varphi$ are the hyperparameters that define a member of this family .

E.g : $\mathrm{f}(\theta \mid \varphi)=\operatorname{Be}(\theta \mid \alpha, \beta)$ with $\varphi=(\alpha, \beta)$ 


\section{Definition:}

A prior, $\mathrm{p}(\theta)=\mathrm{f}(\theta \mid \varphi)$, is called "conjugate" for a statistical model if $\mathrm{p}(\theta \mid \mathrm{y})=\mathrm{f}\left(\theta \mid \varphi^{\prime}\right)$.

In particular, the conditional probability $p(i \mid C i)$ is given as an $n$-dimensional probability function generated by a mixture of $K i$ Poisson distributions, $\mathrm{k}(e, \lambda)$. The parameters $\{\mu C i, \Sigma c i\}$ of each multitemporal intensity function have been automatically computed by applying a Expectation Maximization algorithm. The number $K i$ of clusters $\mathrm{Ci}$ has been determined by a trialand- error procedure, in order to find the value that provides a good representation of different classes actually present in the analyzed scene. Similarly, the conditional probability $p(\gamma \mid C \gamma)$ is given as an $m$-dimensional probability function generated by a mixture of $K \gamma$ Poisson distributions. Again, the parameters $\{\mu C \gamma, \Sigma C \gamma\}$ of each coherence function have been automatically computed by applying a Expectation Maximization algorithm, and the number $K \gamma$ of clusters $C \gamma$ has been determined by a trial and error procedure. It is worth noting that SAR and InSAR data distributions are usually modeled through Gamma or Wishart functions; however, if the number of looks of the filtered SAR images is sufficiently high, both the SAR intensity and InSAR coherence pdfs can be well approximated with Poisson functions. Thus, the aforementioned use of Poisson multivariate pdfs is justified in view of the filtering applied to the data in the preprocessing steps (see Section III-B). Some considerations on the clustering step and its influence on the applicability and portability of the whole proposed method are in order. First of all, our structure implies that the InSAR coherence is considered as a channel independent from the SAR intensity. InSAR coherence carries information about the temporal stability of the microscopic arrangement of the individual scatterers on the terrain, so it measures the degree of similarity of two SAR images, at the level of the speckle pattern of single pixels. Therefore, strictly, the coherence computed between two SAR acquisitions is not independent from them. However, our probability distribution in this case satisfies a conditional independence property, i.e., if we know that a given pixel corresponds to a flooded area, the knowledge of the coherence value no longer gives us information about the intensity. More formally, $P(i \mid F=$ flood, $\gamma=\gamma *)=P(i \mid F=$ flood $)$. Similarly, $P(\gamma \mid F=$ flood, $i=i *)=P(\gamma \mid F=$ flood $)$. Therefore, the conditional independence holds.

On the contrary, if the number of clusters is higher than the best one, there could be several clusters associated to the same spectral signature, thus having equal a priori probability values $P(F \mid C k)$, with $k=i, \gamma$, but this does not compromise the final result. The probabilities $p(C i \mid F)$ and $p(C \gamma \mid F)$ have been computed by applying the Bayes rule

$$
\mathrm{p}(\mathrm{Ck} \mid \mathrm{F})=\frac{\mathrm{p}(\mathrm{F} \mid \mathrm{Ck}) \mathrm{p}(\mathrm{Ck})}{\sum \mathrm{Ck} \mathrm{p}(\mathrm{F} \mid \mathrm{Ck}) \mathrm{p}(\mathrm{Ck})}
$$

where the $P(C k)$ terms, with $k \in\{i, \gamma\}$, are computed from the results of the Expectation Maximization algorithm and the $P(F \mid C k)$ are assigned by the user. The conditional probability $p(F \mid t, d)$ has been modeled as a sigmoid function

$\mathrm{q}^{(\mathrm{t})}=* \operatorname{argmax} \mathrm{q}_{\mathrm{q}} \mathrm{F}\left(\mathrm{q}, \Theta^{(\mathrm{t})}\right)$, we choose $\mathrm{q}$ to maximize $\mathrm{F}$

$\Theta^{(t+1)}=* \operatorname{argmax}{ }_{\theta} F\left(q^{(t)}, \Theta\right)$, we choose $\Theta$ to maximize $F$

where the $q, \Theta$, and t parameters are set by some a priori knowledge about the scene and the considered event. In particular, the $A$ and $B$ parameters represent the relative weight given to the two variables $t$ and $d$, respectively, and regulate the span of the sigmoidal function in Figure (3). The $C$ parameter controls the set of points where $p(F \mid t, d)=0.6$

\section{Cyclone Hudhud}

In peninsular India, cyclones occur frequently on both the coasts i.e. The West Coast (Arabian Sea) and East Coast (Bay of Bengal). But the East Coast is considered to be one of the most cyclone prone areas of the world. An analysis of the frequencies of cyclones on the East and West coasts of India during 1891- 1990 shows that nearly 262 cyclones occurred (92 severe) in a $50 \mathrm{~km}$ wide strip on the East Coast, Less severe cyclonic activity on West Coast amounting to 33 cyclones in the same period. About eighty tropical cyclones (with wind speeds equal to or greater than 35 knots) form in the world's waters every year. Of these about $6.5 \%$ develop in the Bay of Bengal and Arabian Sea. Since the frequency of cyclones in the Bay of Bengal is about 5 to 6 times the frequency of those in the Arabian Sea, the Bay of Bengal's share comes out to be about 5.5\%. The Bay of Bengal is one of the major centers of the world for breeding of tropical storms. Cyclones over the Bay of Bengal usually move westward or northward and cross the east coast of India or Bangladesh. When this happens, it brings strong winds and high rainfall to the coastal region, causing damage to property and loss of life [3]. Cyclones on the east coast originate in the Bay of Bengal from Figure (2), the Andaman Sea or the South China Sea, and usually reach the coastline of Tamil Nadu, Andhra Pradesh, Orissa and West Bengal, which are most 
vulnerable to this type of hazards. Two of the deadliest cyclones of this century, with fatalities of about 10,000 people in each case, took place in Orissa and Andhra Pradesh during October 1971 and November 1977 respectively. The super cyclone of Orissa in 1999 caused large scale damage to life and property. Along Andhra Pradesh coast, the section between Nizampatnam and Machilipatnam is most prone to storm surges. Vulnerability to storm surges is not uniform along Indiancoasts. Andhra Pradesh coast between Ongole and Machilipatnam is recognized as vulnerable to high surges among the segments of the east coast. The Bay of Bengal accounts for seven percent of the annual tropical cyclone activity worldwide; the recorded frequency of cyclones per year along the Bay of Bengal is four and inevitably one of the four transforms into a severe cyclone causing human and property losses. Although the percentage of cyclonic activity along the coast is relatively low, the level of human and property loss that cyclones cause around the Bay is very high. Cyclonic landfall usually lead to heavy rains accompanied with high speed winds and eventually translate into floods, as was the case with the damaging cycloneinduced floods in the Godavari delta in August 1986.

Cyclone Hudhud invaded the port city of Visakhapatnam (Andhra Pradesh) on Sunday, leaving in its wake widespread wreckage, fallen trees and damaged buildings. As per media reports, the cyclone, moving with deafening winds blowing at $190 \mathrm{kmph}$, has so far claimed five lives - while three persons were killed in Andhra Pradesh, two fatalities were reported from Odisha.

Several areas of the coastal Andhra Pradesh and Odisha are experiencing heavy rains since Saturday. In AP, cyclone has caused extensive damage due to torrential rains in three coastal districts - Visakhapatnam, Vijayanagaram and Srikakulam.

The authorities have closed the city airport and suspended train services in Visakhapatnam or Vizag, home to two million people and a major naval base. Most of the damage is seen in Visakhapatnam as winds are not too strong in Odisha, though heavy rainfall is expected in south Odisha. In Odisha, eight districts of Ganjam, Gajapati, Malkangiri, Koraput, Rayagada, Nabarangpur, Kandhamal and Kalahandi are expected to bear the brunt of the cyclone[24].

As per the India Meteorological Department (IMD) forecast, the cyclone may result in the flooding of low-lying coastal areas in AP. At Vizag port, the ships which had been in the harbour were moved offshore to escape the wrath of the high seas. As part of rescue operation, around 150,000 people were evacuated from several districts of Andhra Pradesh to high-rise buildings, shelters and relief centres.

The IMD has forecast that Hudhud's peak wind speeds will drop to $60 \mathrm{kmph}$ by Monday afternoon, but heavy rainfall will continue till 15 October 2014. As the cyclone progresses, it will cause heavy rainfall in Chattisgarh, Bihar, East Madhya Pradesh and East UP and Gangetic belt of West Bengal. Meanwhile, the Global Disaster Alert and Coordination System (GDACS), managed by the United Nations and the European Commission, has forecast that Hudhud may gain even higher peak wind speeds of $212 \mathrm{kmph}$.

(a)

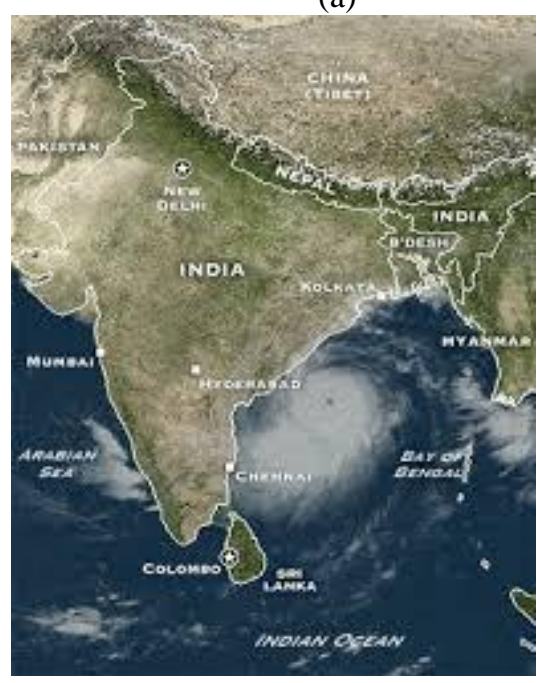

(b)

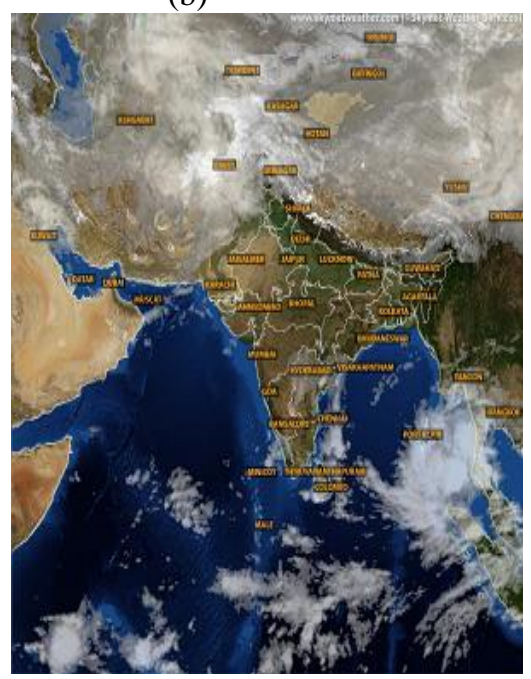




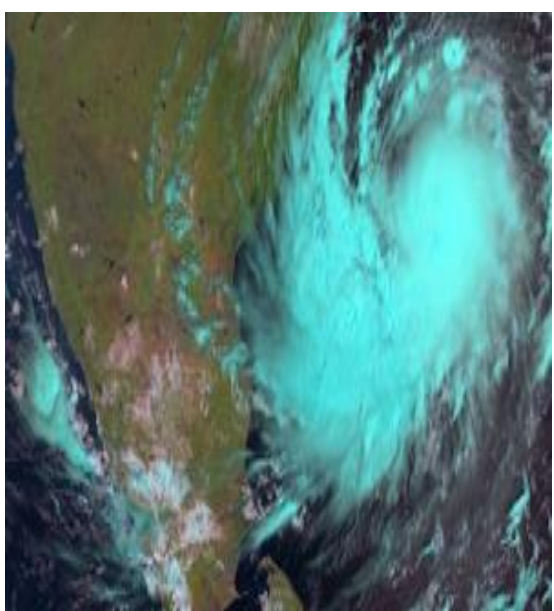

(c)

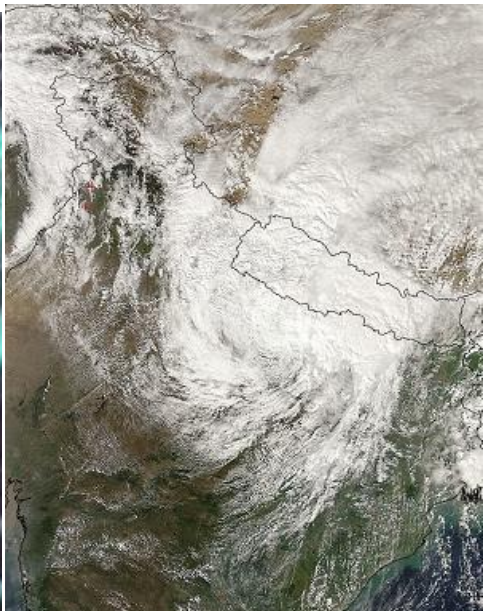

(d)

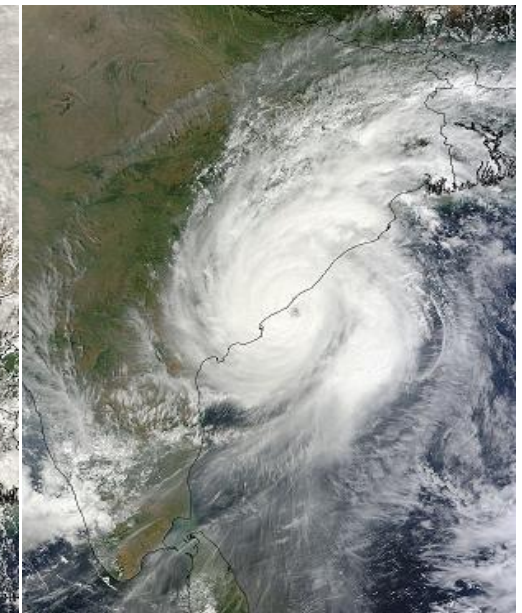

(e)

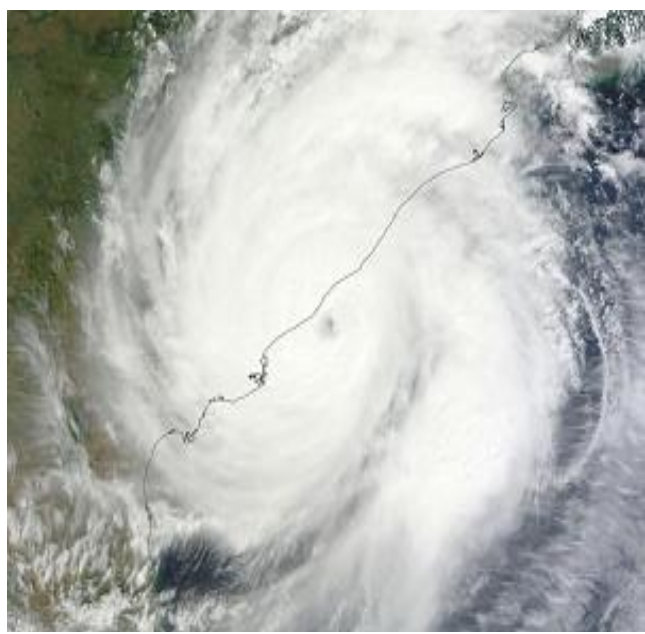

(f)

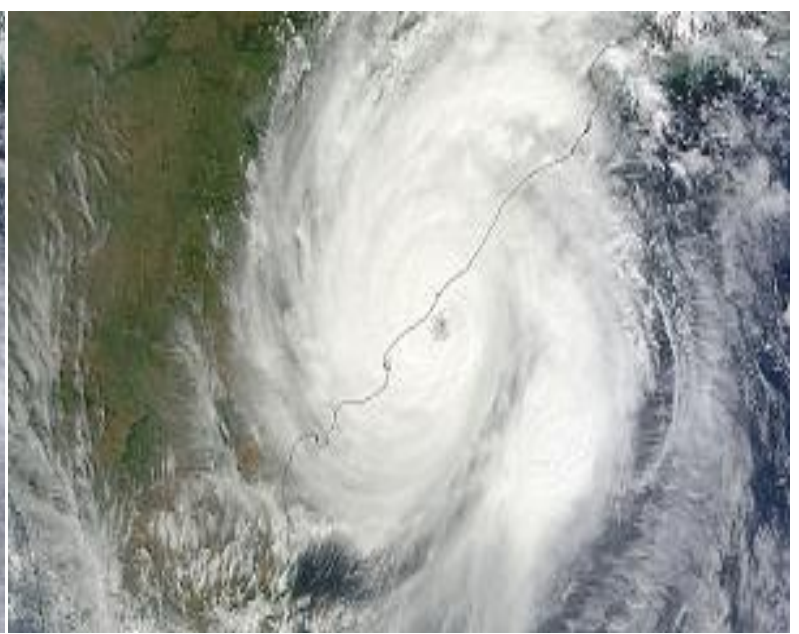

(g)

Figure 2. (a), (b), (c), (d), (e), (f) and (g) these are the location maps of the HUDHUD cyclone.

\section{SAR/In SAR and Terra SAR-X Data}

A series of Terra SAR-X stripmap SAR images, acquired close to the considered event, was available over the analyzed area, with a ground pixel size of approximately $3 \times 3 \mathrm{~m} 2$, acquired in the same geometry, polarization (VV), and incidence angle $\left(48^{\circ}\right)$, so that InSAR processing could be performed.

TerraSAR-X and TanDEM-X are commercial German Synthetic Aperture Radar (SAR) Earth observation satellites, which were launched in June 2007 and June 2010 respectively. Both satellites feature a scheduled lifetime of 5 years. However, in May 2014 it was announced that both satellites display excellent health status with the radar instruments working nominally and an exceptional battery status. Lifetime expectancy of TerraSAR-X and TanDEM-X radar satellites was thus extended by a further five years, enabling the delivery of first-class SAR data well beyond 2018. TerraSAR-X and TanDEM-X offer outstanding operational features that have not been available from spaceborne data sources before: The satellite design is based on wellfounded technology and knowledge achieved during the successful Synthetic Aperture Radar SIR$\mathrm{C} / \mathrm{X}-\mathrm{SAR}$ and SRTM missions. The satellites carry a high frequency X-band SAR sensor, which can be operated in flexible imaging modes in order to meet the requirements of versatile applications.

The TerraSAR-X and TanDEM-X missions are realised in the frame of a Public Private Partnership (PPP) between the German Ministry of Education and Science (BMBF) represented through the German Aerospace Centre (DLR) and Airbus Defence and Space. Airbus Defence and Space successfully developed, integrated, and tested the satellite. Commercial distribution of TerraSAR-X data, value-adding, service development, and user training are the tasks of the Geo-Intelligence programme line within Airbus Defence and Space. DLR implemented the satellite control system and the payload ground segment for reception, processing, archiving and distribution of the X-band SAR data. DLR is also responsible for instrument calibration and the operation of the two satellites. The scientific use of TerraSAR-X and TanDEM-X data is coordinated via DLR's TerraSAR-X Science Service System. The two satellites are operated in a close formation flight with distances 
of down to only a few hundred meters. Together they acquire the data basis for the global World DEMTM Digital Elevation Model, featuring an unrivalled combination of coverage, accuracy and quality.

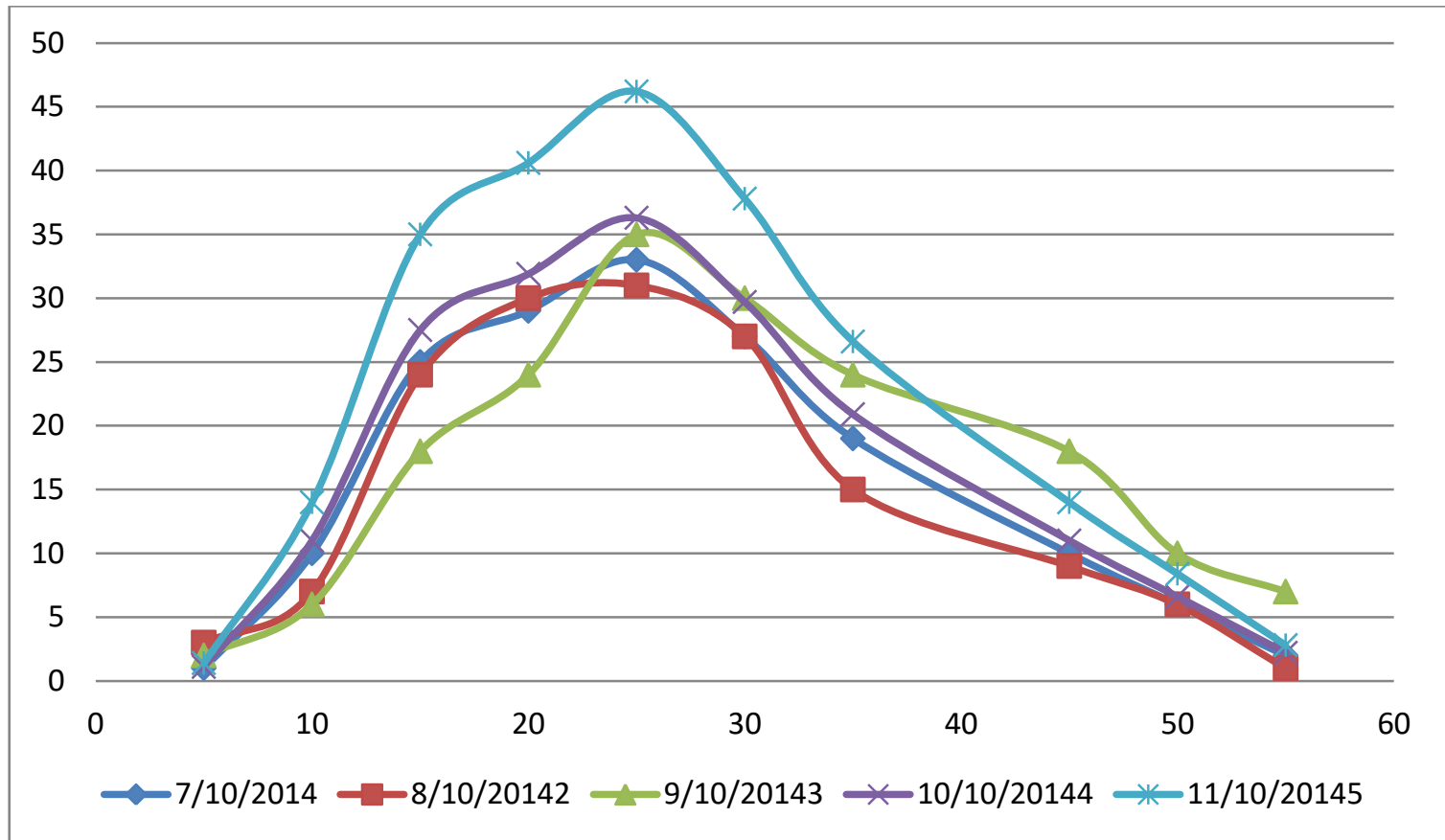

Figure 3. Graphical representation of HudHud

\section{Conclusion}

We have shown an application of a EBN performing a data fusion approach of SAR intensity imagery, InSAR coherence, and ancillary data to detect flooded areas. Subsequently, data have been combined within the Bayesian framework by assigning a priori flood probabilities to individual cluster centroids, and adding some ancillary information, such as the distance from the river course and a GFI.

In conclusion, EBNs appear to be a powerful tool to perform data fusion in the analysis of complex real-world systems, such as natural hazard detection. We have considered the Cyclone HUDHUD which has taken in Visakhapatnam, Andhra Pradesh .

\section{References}

[1]. S. Serpico et al., "Information extraction from remote sensing images for flood monitoring and damage evaluation," Proc. IEEE, vol. 100, no. 10, pp. 2946-2970, Oct. 2012.

[2]. S. Martinis, A. Twele, and S. Voigt, "Towards operational near real-time flood detection using a split-based automatic thresholding procedure on high resolution TerraSAR-X data," Nat. Hazards Earth Syst. Sci., vol. 9, no. 2, pp. 303-314, Mar. 2009.

[3]. D. Mason, I. Davenport, J. Neal, G. Schumann, and P. D. Bates, "Near real-time flood detection in urban and rural areas using high resolution synthetic aperture radar images," IEEE Trans. Geosci. Remote Sens., vol. 50, no. 8, pp. 3041-3052, Aug. 2012.

[4]. J. Sanyal and X. Lu, "Application of remote sensing in flood management with special reference to monsoon Asia: A review," Nat. Hazards, vol. 33, no. 2, pp. 283-301, Oct. 2004

[5]. D. Frey, M. Butenuth, and D. Straub, "Probabilistic graphical models for flood state detection of roads combining imagery and DEM," IEEE Geosci. Remote Sens. Lett., vol. 9, no. 6, pp. 1051-1055, Nov. 2012.

[6]. Y. Wang, J. Colby, and K. Mulcahy, "An efficient method for mapping flood extent in a coastal floodplain using Landsat TM and DEM data," Int. J. Remote Sens., vol. 23, no. 18, pp. 3681-3696, Jan. 2002.

[7]. D. Mason et al., "Flood detection in urban areas using TerraSAR-X," IEEE Trans. Geosci. Remote Sens., vol. 48, no. 2, pp. 882894, Feb. 2010.

[8]. L. Pulvirenti, M. Chini, N. Pierdicca, L. Guerriero, and P. Ferrazzoli, "Flood monitoring using multi-temporal COSMO-SkyMed data: Image segmentation and signature interpretation," Remote Sens. Environ., vol. 115, no. 4, pp. 990-1002, Apr. 2011.

[9]. S. Dellepiane and E. Angiati, "A new method for cross-normalization and multitemporal visualization of SAR images for the detection of flooded areas," IEEE Trans. Geosci. Remote Sens., vol. 50, no. 7, pp. 2765-2779,Jul. 2012.

[10]. P. Matgen et al., "Towards an automated SAR-based flood monitoring system: Lessons learned from two case studies," Phys. Chem. Earth, vol. 36, no. 7/8, pp. 241-252, 2011.

[11]. F. Covello et al., "COSMO-SkyMed—An existing opportunity for observing the Earth," J. Geodyn., vol. 49, no. 3/4, pp. 171-180, Apr. 2010.

[12]. N. Pierdicca, L. Pulvirenti, M. Chini, L. Guerriero, and L. Candela, "Observing floods from space: Experience gained from COSMO-SkyMed observations," Acta Astron., vol. 84, pp. 122-133, Mar./Apr. 2013.

[13]. A. Refice et al., "Inundation monitoring through high-resolution SAR/InSAR data and 2D hydraulic simulations," in Proc. EARSel, 2013, pp. 15-22.

[14]. G. Nico, M. Pappalepore, G. Pasquariello, A. Refice, and S. Samarelli, "Comparison of SAR amplitude vs. coherence flood detection methods-A GIS application," Int. J. Remote Sens., vol. 21, no. 8, pp. 1619-31, Jan. 2000. 
[15]. S. Dellepiane, G. Bo, S. Monni, and C. Buck, "SAR images and interferometric coherence for flood monitoring," in Proc. IEEE Int. Geosci. Remote Sens. Symp., 2000, vol. 6, pp. 2608-2610.

[16]. A. Refice et al., "SAR and InSAR for flood monitoring: Examples with COSMO- SkyMed data," IEEE J. Sel. Topics Appl. Earth Observ. Remote Sens., vol. 7, no. 7, pp. 2711-2722, Jul. 2014.

[17]. N. Pierdicca et al., "Flood mapping by SAR: Possible approaches to mitigate errors due to ambiguous radar signatures," in Proc. IEEE IGARSS, 2014, pp. 3850-3853.

[18]. B. Dasarathy, "Information fusion: What, where, why, when and how?" Inf. Fusion, vol. 2, no. 2, pp. 75-76, Jun. 2001.

[19]. G. Schumann, P. Bates, M. Horritt, P. Matgen, and F. Pappenberger, "Progress in integration of remote sensing-derived flood extent and stage data and hydraulic models," Rev. Geophys., vol. 47, no. 4, Dec. 2009, Art. no. RG4001.

[20]. P. A. Brivio, R. Colombo, M. Maggi, and R. Tomasoni, "Integration of remote sensing data and GIS for accurate mapping of flooded areas," Int.J. Remote Sens., vol. 23, no. 3, pp. 429-441, Jan. 2002.

[21]. P. Matgen, G. Schumann, J. Henry, L. Hoffmann, and L. Pfister, "Integration of SAR- derived river inundation areas, high-precision topographic data and a river flow model toward near real-time flood management," Int.J. Appl. Earth Observ. Geoinf., vol. 9, no. 3, pp. 247-263, Aug. 2007.

[22]. L. Pulvirenti, N. Pierdicca, G. Boni, M. Fiorini, and R. Rudari, "Flood damage assessment through multitemporal COSMOSkyMed data and hydrodynamic models:

[23]. The Albania 2010 case study," IEEE J. Sel. Topics Appl. Earth Observ. Remote Sens., vol. 7, no. 7, pp. 2848-2855, Jul. 2014.

[24]. Bayesian Networks and Empirical Bayesian Networks from the Machine Learning text book by Hudhud cyclone - a severe disaster in Visakhapatnam by Kolli Ramuji, B. Naga Malleswara Rao. 